\title{
SUMMARY OF THE LITERATURE RELATIVE TO THE FORMATION OF FILM ON POLISHED GLASS SURFACES
}

\author{
By George W. Morey
}

The stability of polished glass surfaces is of prime importance in the design and manufacture of optical instruments, and all factors affecting their stability are worthy of careful consideration. Some glasses are inherently inferior in their resistance to the corrosive action of water and weak acids, and such glasses are to be avoided whenever possible. Experience has shown the relative reliability of different glass types and glass compositions, and tests have been devised which enable the optician to avoid actually inferior types. ${ }^{1}$ But glasses possessing a high degree of resistance to such "weathering" action often give trouble by becoming covered with a coating resembling that produced by the weathering of an inferior glass, but actually due to entirely different causes. This particular type of coating has been called "film," and the following report summarizes the information contained in the literature on the formation, appearance, cause, and prevention of "film" on polished glass surfaces.

The phenomenon in question is thus described by Ryland:2 "In every type of enclosed optical instrument, instances, more or less numerous, of film will be found. By film is meant the coating of lens and prism surfaces with what is apparently a thin deposit of moisture. This has caused much trouble and annoyance, many instruments being rendered quite useless until taken apart and cleaned. The film appears to have several characteristics. It apparently consists of a series of globules of moisture, forming patterns on the surface similar to those made by cleaning with a linen rag which has seen much service. Often a series of larger globules will occur, each surrounded by a clear space.

${ }^{1}$ G. W. Morey, J. Soc., Glass Technology, 6, p. 20-30; 1922.

${ }^{2}$ H. S. Ryland, Trans. Opt. Soc., 19, p. 178-83; 1918-19. 
Parts of a surface under pressure from mounts will be clear for a small space surrounding the points of contact, the clear space being fringed by a denser deposit. Prisms will often show a distinct pattern of the seat, the part covered by metal having a fairly dense deposit, a fairly clear space occurring where the surface is free, becoming again more dense away from the metal. A cut, scratch or hole in the surface is generally surrounded by a clear space, beyond which the deposit is again more dense.

"If a drop of water from a pipette is allowed to run over a filmed surface, it will leave a track which is quite free from any trace of scum. Microscopic examination shows no injury to the surface after filming. Instruments such as telescopes, in which the air is occasionally changed, very seldom film. When they do it is generally the case that they have been out of service for some time."

The above description of filming agrees well with that of other writers. That, even in cases in which the film appears uniform to the unaided eye, it actually consists of tiny discrete drops, is stated by Ryland (loc. cit., p. 183) and by Jones. ${ }^{3}$ The tendency of film to form on reticules ("graticules") and similar etched surfaces is often mentioned; ${ }^{4}$ an excellent photomicrograph of such a deposit in a reticule from a prism binocular is given by Martin and Griffith. ${ }^{5}$ That such deposits are usually found in instruments which after assembly are made air and water tight as opposed to those in which there is opportunity for circulation of air is brought out by Jones, by Ryland and by Beck. The latter also states that "The Admiralty informed him that they have never met with it (film) inside submarine periscopes which are hermetically sealed and filled with dry air. He was told that it had not been met with in the Aldis unit sight when properly sealed, except in some experimental cases when a coating of

${ }^{3}$ Remarks on the filming of glass. By H. S. Jones of the Inspection Dept., D. I. O. S. Report submitted by Lt. Col. A. C. Williams, Dept. of Scientific and Industrial Research, Standing Committee on Glass and Optical Instruments.

${ }^{4}$ Discussion on the filming of glass held by the British Optical Instrument and Manufacturers Association.

${ }^{5}$ L. C. Martins and C. H. Griffiths. Trans. Opt. Soc. London, 20, p. 135-54; 1920. 
glycerine had been placed on the internal surfaces to pick up floating dust particles." Martin and Griffiths state that film is most often found in reticules, next in field lenses of eye pieces, next in prisms.

It follows from the above that in enclosed optical instruments a deposit, consisting of more or less discrete drops, tends to form on the glass surfaces, most especially on reticules. It also follows, e.g., from the data furnished by Beck, that such film is not met with even in enclosed instruments, when such instruments are assembled with great care, as is the case with periscopes. The important conclusion that the film is not a permanent attack of the glass surface, but on the contrary the original surface appears unchanged even when examined under the microscope after washing off the film with distilled water, is confirmed by Martin and Griffiths. The latter report: "As far, at least, as the globular deposit is concerned, the surface of the glass shows no trace of corrosion, when examined under the microscope, after the deposit of film has been washed off."

The cause of film has not yet been established. Two factors, however, seem to be necessary; the presence of water (moisture) and the presence of dirt or grease, especially the latter. French, ${ }^{6}$ Chalmers, ${ }^{7}$ and Wright ${ }^{8}$ emphasize this point. Ryland writes, "A number of experiments have been tried in order to determine the cause of film. Certain types of black, notably the japan black and those containing pitch or bitumen, almost always cause film; aluminum machined and not cleaned afterwards; a small trace of animal matter or beeswax in the closed space will give trouble." Martin and Griffiths state that "moisture alone has no affect in producing the globular deposit" and that "the presence of lubricants does not produce globular deposit nor disintegration of surfaces unless moisture is present." The consensus of opinion is that, irrespective of the stability of the glass, elimination of dirt, grease and moisture are essential to the prevention of film.

' See discussion of the paper by Ryland, loc. cit.

${ }^{7}$ See discussion of the paper by Martin and Griffiths, loc. cit.

8 "The Manufacture of Optical Glass and of Optical Systems," Lieut. Col. F. E. Wright. Ordnance Dept. Document No. 2037. 
Ryland, after outlining precautions to be taken in cleaning, writes, "The use of these precautions will apparently make even poor glass remain free from film." French, Beck, Chalmers, Jones and Wright all agree as to the imperative necessity of meticulous cleanliness in assembly. Jones states that, "Generally speaking, it may be said that those firms who had taken the greatest care with the cleaning to ensure absolute cleanliness had the least trouble with film, whilst those who had taken fewest precautions to ensure cleanliness had most trouble." He also cites this case: "One firm who have had very considerable trouble from film, noticed that not a single instrument in which lenses etc. had been cleaned by the foreman of the assembly department had been rejected for film, and that the ordinary cleaning methods were used, i.e., without special precautions."

The nature of the glass, however, is probably not without importance. While Ryland asserts that proper precautions will prevent film on even poor glass, he states that "Different glasses however appear to have different susceptibility to film; the most troublesome glass I have found to be one made of borosilicate crown." In the discussion on filming held by the British Optical Instrument Manufacturers Association, it was brought out that "generally speaking, glasses showing the greatest affinity for water give the most trouble," though in this connection it should be noted that borosilicate crown, designated by Ryland as the most troublesome, is not especially susceptible to weathering. In addition to laying stress on cleanliness in assembly it is stated that "Care should be taken in the choice of glass. Hygroscopic glasses are to be avoided." Martin and Griffiths in a series of experiments on the formation of film, were unable to detect any difference between ordinary crown and light flint. On the other hand they state that no film had ever been observed on reticules made by a certain German firm, who used a light barium flint, Jena $\mathrm{O} 463$. The evidence collected by Jones on this point is mainly negative, with the exception of an instance cited in which, "One firm had a lot of trouble with the graticulated discs of prismatic binoculars owing to the formation of globular deposits. This has been entirely overcome by simply changing from plate 
glass to baryta light flint, without alteration of methods of cleaning." This latter case is probably one in which an actually poor glass had been used, as opposed to the majority of cases in which film was formed on fairly resistant glass, as a result of careless cleaning. It shows the necessity of choosing a good type of glass for the manufacture of such parts as reticules, though the light barium flint is probably not superior to many other glasses in common use.

Following are the precautions enumerated by Ryland as a complete preventative of filming:

1. No japan or bitumen black to be used on interior surfaces.

2. No pitch or beeswax to be used within the case.

3. All interior surfaces must be thoroughly cleaned and free from all animal matter such as finger marks. In the case of aluminum or other porus metal, heat treatment is necessary after machining to remove all trace of grease.

4. The lenses and prisms after ordinary cleaning to be dipped in hot running water with a pair of tweezers and wiped dry with clean linen (see 5 and 7 ) without being touched by the fingers again.

5. After washing the linen used for cleaning purposes, it must be well rinsed in hot water until all trace of soap is gone.

6. Dust must be removed with a clean camels hair brush and not by blowing.

7. The linen used for cleaning must be used in such a way that no part of it which has been touched by the fingers is brought into contact with the lens or prism surfaces.

8. All surfaces must be well polished and free from "orange peel" effect. If the pitch polisher leaves a trace of scum, a small quantity of glacial acetic acid added to the pitch when melted will prevent it."

While all writers do not agree that the above precautions will absolutely prevent film (Martin and Griffiths, Beck) there seems to be no doubt as to their being efficacious in most cases. In regard to the individual precautions, in some cases japan or bitumen do not appear to be at fault: "The black used by some American firms has not been found to be satisfactory in some instances, 
and improvement had resulted in the use of a black with a reliable composition" (Jones). That precaution No. 3 is essential appears to be without dispute. Precaution No. 4, dipping lenses, after ordinary cleaning, is recommended by Martin and Griffiths, whose experiments show clearly its efficiency.

From the facts enumerated, the following conclusions can be drawn with some degree of assurance.

1. Filming, i.e., the formation of more or less isolated drops on the polished surfaces of glass instruments, is a result of the deposition of water contaminated by greasy matter.

2. Both water and grease are essential to the formation of film, and hence the utmost care must be taken to thoroughly clean the glass surfaces themselves, to ensure that no moisture or grease, or material which may yield moisture or grease, is contained in the space within which the glass surfaces are confined.

3. The glass should be of good quality, at least as good as Class 3 as determined by the iodeosine test.

Geophysical Laboratory,

Carnegie Institution of Washington, JULY, 1922. 